\title{
PERÍCIA EM FURTO DE ENERGIA ELÉTRICA EM INSTALAÇÕES RESIDENCIAIS
}

Marcelo de Jesus Rodrigues da Nóbrega

Pós Doutor em Engenharia pela Universidade Estadual do Rio de Janeiro (UERJ)

Rio de Janeiro, RJ, Brasil.

Perito de Engenharia no Tribunal de Justiça do Estado do Rio de Janeiro (TJRJ)

Rio de Janeiro, RJ, Brasil.

engmarcelocefet@terra.com.br

\section{RESUMO}

A demanda crescente de processos judiciais que envolvem furtos de energia elétrica, em especial em instalações residenciais, gera, na mesma proporção, laudos periciais e pareceres técnicos. 0 principal objetivo deste artigo é apresentar as principias referências que devem ser utilizadas na elaboração de um laudo pericial para casos de furto de energia em residências, e ainda relacionar os aspectos técnicos da área de engenharia elétrica as ferramentas computacionais disponíveis. Realizou-se uma revisão da literatura que envolve a consulta principalmente a legislação aplicável, Resolução ANEEL 414/2010 e Lei Federal no 13.105, além de obras clássicas da engenharia e perícia judicial, como CREDER (2008), FIKER (2011), MAIA (2019) e SERTÃ JUNIOR (2019). Concluiuse que há grande importância sobre o conhecimento teórico, aliando, porém, a prática metodológica que deve ser obrigatoriamente seguida, sob pena de não homologação ou impugnação do laudo pericial.

Palavras-chave: Engenharia. Perícia. Elétrica. Energia.

\section{EXPERTISE ON ELECTRIC POWER THEFT IN RESIDENTIAL INSTALLATIONS}

\begin{abstract}
The increasing demand for lawsuits that involve theft of electric power, especially in residential installations, generate to the same extent, expert reports and technical advice. The main purpose of this article is to present the main references that should be used in the elaboration of an expert report for cases of electric power theft in residences, and to relate the technical aspects of the electrical engineering area to the available computational tools. A literature review was conducted involving consultation of applicable legislation, ANEEL Resolution 414/2010 and Federal Law No. 13,105 , in addition to classic engineering works and judicial expertise, such as CREDER (2008), FIKER (2011), MAIA (2019). ) and SERTÃ JUNIOR (2019). It was concluded that there is great importance about the theoretical knowledge, combining, however, the methodological practice that must be followed, under penalty of will not be homologate or impugn of the expert report.
\end{abstract}

Keywords: Engineering. Expertise. Electric Energy.

\section{INTRODUÇÃO}

O presente trabalho tem por objetivo realizar uma revisão da literatura e também da legislação, no que se refere ao trabalho do perito judicial, em especial, nos casos de Perícia em Furtos de Energia Elétrica. A justificativa deste estudo está na falta de uma literatura que trate de 
forma sistemática, apresentando os fundamentos básicos de eletricidade envolvidos, normas técnicas, legislações, simuladores de demanda de consumo de energia, equações típicas e abordagem de atribuições legais estabelecidas pelo conselho classe, no âmbito da engenharia.

Desta forma, foi realizada uma a pesquisa bibliográfica aplicada ao tema incluindo também normas e legislações aplicadas à temática do trabalho. Obras clássicas auxiliaram na elaboração do presente estudo, tais como: Maia (2019), Creder (2008), Fiker (2011), Sertã Júnior (2019), além de legislações e normas: NBR 5410 (2004) da Associação Brasileira de Normas Técnicas - ABNT; Resolução Normativa 414/2010 da ANEEL; Lei Federal no 13.105/2015 e Lei Federal no 6.496.1977.

\section{DESENVOLVIMENTO}

\subsection{Fundamentos de Engenharia Elétrica aplicado a Perícia}

A energia elétrica atualmente pode ser gerada de diversas maneiras, por exemplo: fonte solar, fonte radioativa, hidroelétrica, sistemas de geradores por queima de diversos combustíveis, dentre outras. No Brasil a matriz é hidráulica. A Figura 1 apresenta, em síntese, todo o processo de geração, transmissão, distribuição de energia, chegando assim, até consumidor final.

Figura 1 - Sistema de geração, transmissão, distribuição e consumo de energia para

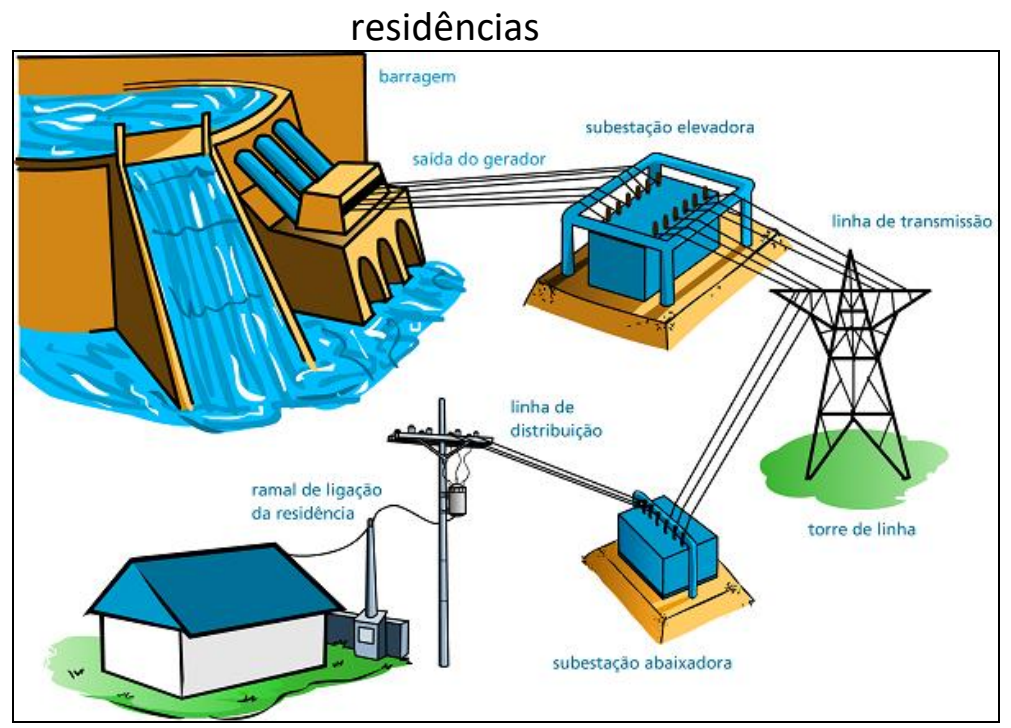

Fonte: (https://www.mundodaeletrica.com.br/um-pouco-mais-sobre-o-sistema-eletrico-de-potencia-sep/)

A Figura 2, ilustra um esquema típico de ligação elétrica do poste da rede da concessionária até o medidor de consumo em residência. Importante observar a ligação feita em residência do mesmo lado que o poste da rede e da residência do lado oposto, neste caso existindo a necessidade de um poste auxiliar. 
Figura 2 - Esquema de ligação elétrica do poste até o medidor de consumo em residência

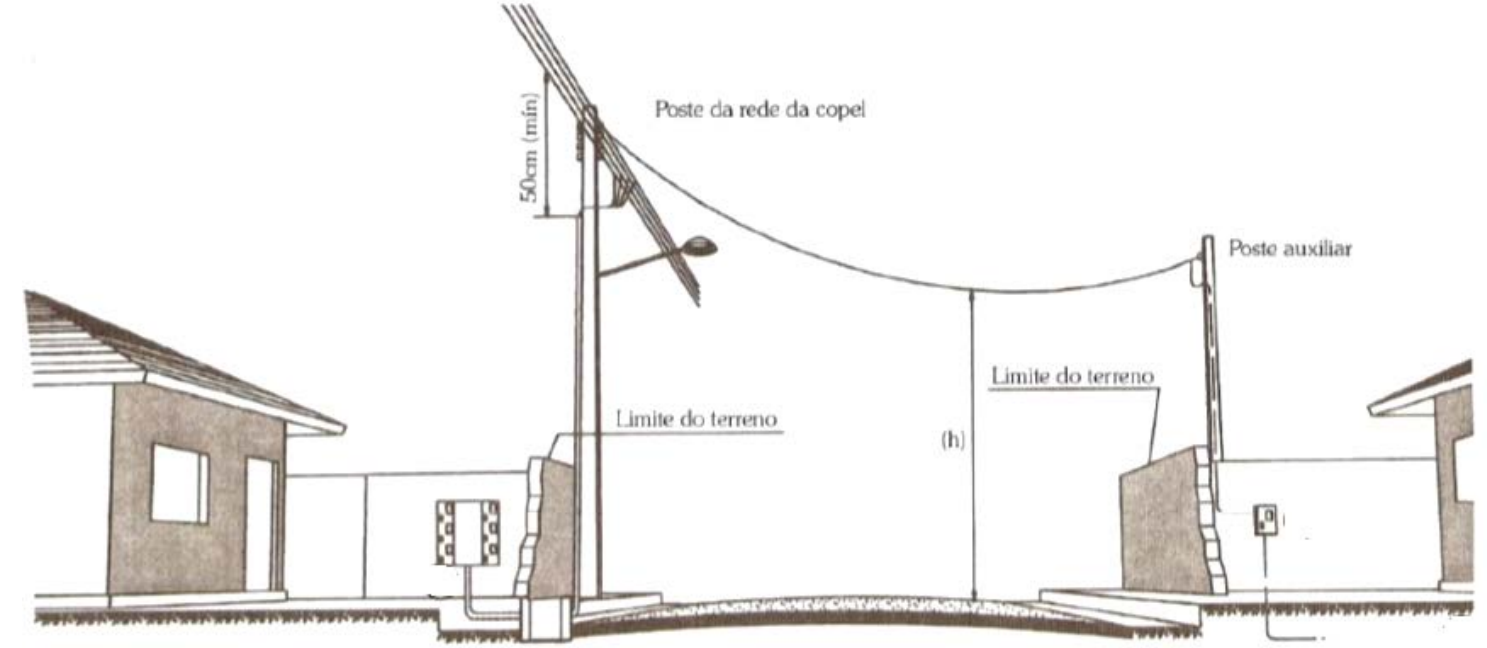

Fonte: (Material didático do Curso de Especialização em Engenharia Elétrica - Universidade Cândido Mendes, 2019).

A concessionária local deve fornecer o padrão de entrada, conforme CREDER (2008). A empresa LIGHT disponibiliza em seu site na internet o RECON-BT.2019, sendo mostrado nas Figuras 3, 4 e 5 uma reprodução parcial dos detalhes técnicos a serem observados em um instalação residencial em baixa tensão.

Figura 3 - Ligação no padrão da concessionária

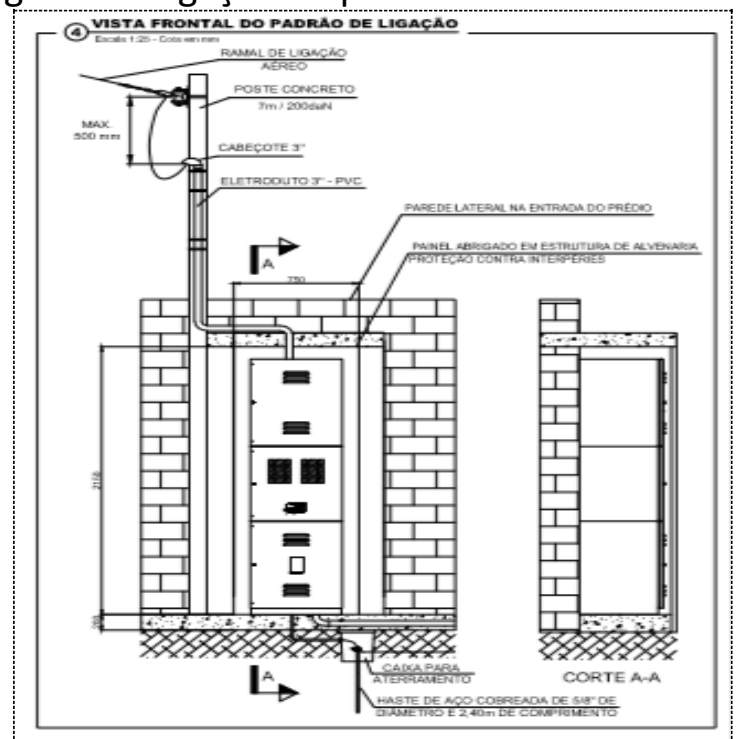

Fonte: (RECON-BT, 2019). 
Figura 4 - Ligação de medidor trifásico até o quadro de distribuição
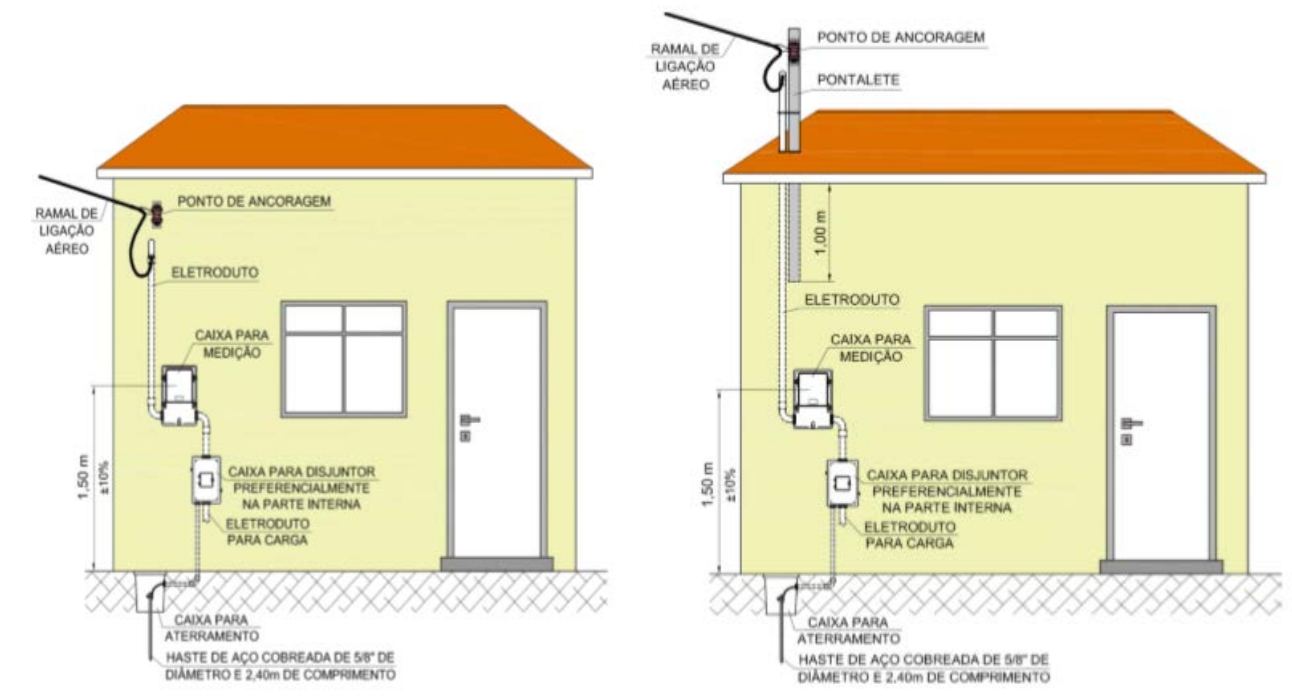

Fonte: (RECON-BT, 2019).

A Figura 5, apresenta os medidores de consumo de energia elétrica a saber: monofásico (fase/neutro); bifásico (fase/fase/neutro) e trifásico (fase/fase/fase/neutro).

Figura 5 - Ligação de medidores até o quadro de distribuição

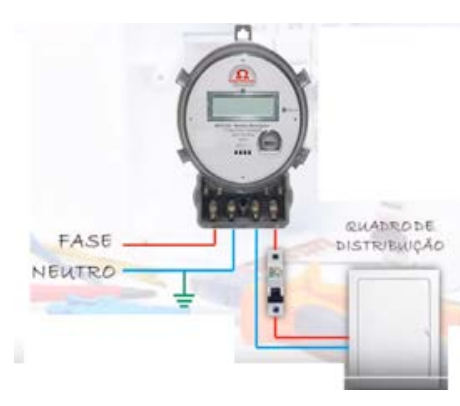

(a) Medidor monofásico

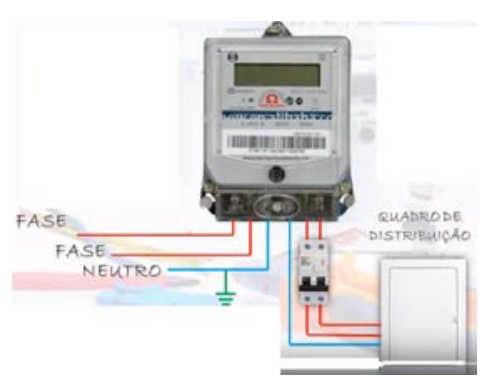

(b) Medidor bifásico

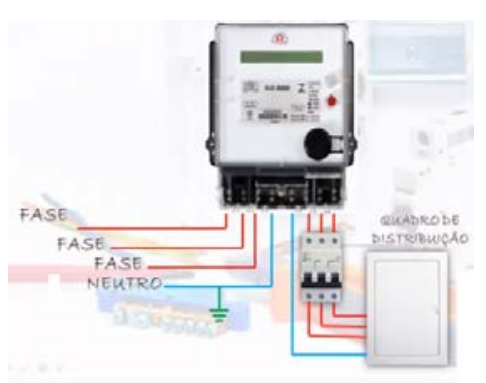

(c) Medidor trifásico

Fonte: (http://omegatreinamentos.coursify.me/courses/)

\subsection{Habilitação Legal}

No âmbito dos profissionais da área de engenharia, em análise da resolução Confea no 218/1973 que discrimina atividades das diferentes modalidades profissionais da Engenharia, verifica-se que as mais aderentes a realização de Perícia e Assistência Técnica em furto de energia elétrica são os engenheiros Mecânicos, Eletricistas e Civis.

No artigo 1 으 da referida resolução, ficam designadas as seguintes atividades:

$$
\text { (...) Atividade } 06 \text { - Vistoria, perícia, avaliação, arbitramento, laudo e parecer técnico. }
$$

No artigo 7으, compete ao engenheiro civil: 
I - o desempenho das atividades 01 a 18 do artigo $1^{\circ}$ desta Resolução, referentes a edificações, estradas, pistas de rolamentos e aeroportos; sistema de transportes, de abastecimento de água e de saneamento; portos, rios, canais, barragens e diques; drenagem e irrigação; pontes e grandes estruturas; seus serviços afins e correlatos.

Compete ao engenheiro eletricista, de acordo com o artigo 9o:

I - o desempenho das atividades 01 a 18 do artigo 10 desta Resolução, referentes a materiais elétricos e eletrônicos; equipamentos eletrônicos em geral; sistemas de comunicação e telecomunicações; sistemas de medição e controle elétrico e eletrônico; seus serviços afins e correlatos.

Já no artigo 12 é de competência do engenheiro mecânico:

I - o desempenho das atividades 01 a 18 do artigo 1 ㅇ desta Resolução, referentes a processos mecânicos, máquinas em geral; instalações industriais e mecânicas; equipamentos mecânicos e eletromecânicos; veículos automotores; sistemas de produção de transmissão e de utilização do calor; sistemas de refrigeração e de ar condicionado; seus serviços afins e correlatos.

Em trabalhos periciais deve-se, obrigatoriamente, ser realizada a Anotação de Responsabilidade Técnica (ART) junto ao sistema CREA/Confea, uma vez que foi regulamentado pela Lei Federal no 6.496/1977.

\subsection{Metodologia em Perícia Judicial}

A metodologia é um item obrigatório em laudos periciais, pois foi estabelecido pela Lei Federal no 13.105/2015. Em muitos laudos, infelizmente, este item não é verificado, devendo assim tornar-se de pronto o laudo nulo, uma vez que não se curva a legislação vigente. Em termos de referenciais metodológicos, Maia (2019), Sertã Junior (2019) e Fiker (2011) definem alguns princípios norteadores para uma perícia de furto de energia, sendo eles:

I - Princípio da Observação;

II - Princípio da Análise;

III - Princípio da Interpretação;

IV - Princípio da Descrição;

V - Princípio da Documentação.

Considerando a disponibilidade e acesso na internet, a seguir é apresentada uma ementa de um processo judicial a nível apelação. No caso concreto, trata-se de constatação de desvio de 
energia no relógio medidor e devendo ainda observar que o furto de energia gerou este processo de cunho criminal.

0040484-93.2012.8.19.0014 - APELAÇÃO 1a Ementa Des(a). FLAVIO MARCELO DE AZEVEDO HORTA FERNANDES - Julgamento: 26/06/2018 - SEGUNDA CÂMARA CRIMINAL APELAÇÃO CRIMINAL. FURTO DE ENERGIA ELÉTRICA. Art. 155, §§ 3 e 4ำ II, do Código Penal. Alegação de violação ao Princípio da Identidade Física do Juiz. Não há que se falar em vinculação decorrente do Princípio da Identidade Física do Juiz em razão da remoção da magistrada para outra Vara Criminal. Constatada a fraude no relógio medidor e evidenciada a redução de consumo, fica clara a existência do "animus furandi", configurando-se o delito com a mera utilização de qualquer aparelho eletrodoméstico. Qualificadora de fraude configurada. Ligação clandestina inteiramente escondida embaixo da caixa de energia elétrica, de forma a impedir a constatação da irregularidade pela Ampla. Impossibilidade de concessão do benefício previsto no art. 89 da Lei no 9099/95. Pedido de isenção de custas processuais que deve ser deduzido junto ao Juízo da Execução, nos termos da Súmula no 74-TJRJ. RECURSO DEFENSIVO A QUE SE NEGA PROVIMENTO.

Fonte: processo 0040484-93.2012.8.19.0014

De acordo com a legislação em vigor, a concessionária deve adotar procedimentos técnicos e administrativos, conforme Resolução ANEEL 414/2010, especialmente na emissão do Termo de Ocorrência e Inspeção (TOI). Em diversos processos judiciais verifica-se a falta de habilidade no atendimento a tais aspectos, tornando, assim, o referido TOI nulo.

\subsection{Determinação da Demanda}

Muitos processos judiciais versando sobre furto de energia elétrica, têm como ponto controverso a determinação da demanda. Neste sentido é importante deixar bem claro que a potência ou carga instalada, é o somatório das potências nominais dos equipamentos ligados em uma instalação do consumidor à rede de energia elétrica da concessionária. Cabendo ressaltar que o fabricante de cada equipamento fornece a potência nominal.

Já a demanda de utilização ou também conhecida como provável demanda, é o somatório das potências nominais de todos os aparelhos elétricos que funcionam simultaneamente, conforme ilustrado na Figura 6, observa-se que a demanda é função temporal.

Figura 6 - Curva característica de consumo residencial

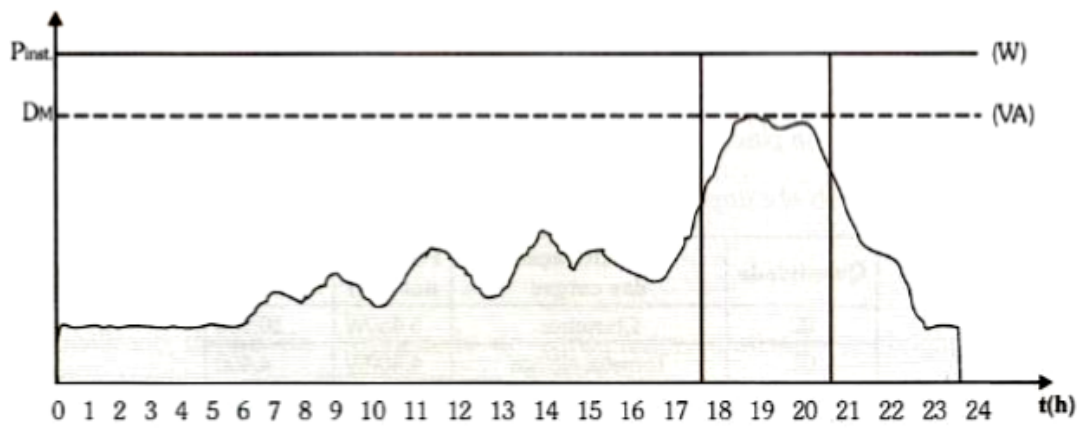

Fonte: (MATERIAL DIDÁTICO, 2019). 
A equação (1) apresenta o consumo, expresso em kWh, de cada um dos equipamentos de uma instalação, sendo de amplo uso em conta de energia das concessionárias.

$$
\begin{aligned}
C_{i} & =\frac{P \cdot h \cdot d}{1000} \\
C & =\sum C_{i}
\end{aligned}
$$

Onde:

Ci: consumo de cada equipamento (kWh);

$P$ : potência do equipamento (W);

$h$ : tempo de uso do equipamento em horas por dia ( $h / d i a)$;

$d$ : número de dias em uso do equipamento (dias);

$C$ : consumo total dos equipamentos (kWh);

Aplicando a verificação dimensional em unidades do sistema internacional, tem-se a equação (3):

$$
[C]=\frac{[W],[\mathrm{h} / \mathrm{dia}],[\text { dia }]}{1000}=[\boldsymbol{k W} \cdot \boldsymbol{h}]
$$

Desta forma, diversas concessionárias de energia elétrica já dispõem de simuladores online, como é o caso da Light no Rio de Janeiro (http://www.light.com.br/pararesidencias/Simuladores/consumo.aspx) e a Cemig (https://www.cemig.com.br/ptbr/atendimento/Paginas/simulador_de_consumo.aspx) em Minas Gerais.

A Tabela 1, ilustra uma simulação de uma residência que possui: três lâmpadas fluorescentes de $11 \mathrm{~W}$, três lâmpadas fluorescentes de $15 \mathrm{~W}$, três lâmpadas fluorescentes de 23 W, uma lava roupas, uma TV 29", sendo simulado neste trabalho meramente para fins didáticos. Estes simuladores são de grande valia para perícias em furtos de energia, especialmente por sua facilidade de operação e disponibilidade.

Cabe ressaltar que são adotados valores usuais em termos de potência e tempo diário de uso para cada tipologia de equipamento. Para o caso da potência, uma análise mais verossímil, deve-se consultar o manual do fabricante do equipamento. No que tange horas diárias de uso, as estações do ano permitem uma variação desta estimativa a maior ou a menor. Assim, o perito deve estar ciente da dispersão associada ao valor médio estimado, fornecido por tais programas de concessionárias de energia. 
Tabela 1 - Simulação do consumo de energia

\begin{tabular}{|c|c|c|c|c|c|c|}
\hline \multicolumn{7}{|c|}{ ENTRETENIMENTO } \\
\hline Aparelho & Quant. & Potência (W) & Tempo & Unid. Tempo & Dias no Mês & Total kWh \\
\hline TV em cores - 29" & 1 & 110 & 5 & Horas & 30 & 16.5 \\
\hline \multicolumn{7}{|c|}{ Total: 17 kWh/mês } \\
\hline \multicolumn{7}{|c|}{ LAVANDERIA/LIMPEZA } \\
\hline Aparelho & Quant. & Potência (W) & Tempo & Unid. Tempo & Dias no Mês & Total kWh \\
\hline Lavadora de roupas & 1 & 500 & 1 & Horas & 12 & 6 \\
\hline \multicolumn{7}{|c|}{ Total: 6 kWh/mês } \\
\hline \multicolumn{7}{|c|}{ ESCRITÓRIO } \\
\hline Aparelho & Quant. & Potência (W) & Tempo & Unid. Tempo & Dias no Mês & Total kWh \\
\hline Microcomputador & 1 & 80 & 2 & Horas & 30 & 4.8 \\
\hline \multicolumn{7}{|c|}{ Total: 5 kWh/mês } \\
\hline \multicolumn{7}{|c|}{ ILUMINAÇÃO } \\
\hline Aparelho & Quant. & Potência (W) & Tempo & Unid. Tempo & Dias no Mês & Total kWh \\
\hline Lâmpada fluoresc. compacta - $11 \mathrm{~W}$ & 3 & 11 & 5 & Horas & 30 & 4.95 \\
\hline Lâmpada fluoresc. compacta - 15 W & 3 & 15 & 5 & Horas & 30 & 6.75 \\
\hline Lâmpada fluoresc. compacta - $23 \mathrm{~W}$ & 3 & 23 & 5 & Horas & 30 & 10.35 \\
\hline
\end{tabular}

Total: 22 kWh/mês

Fonte: (http://www.light.com.br/para-residencias/Simuladores/consumo.aspx)

\section{CONCLUSÃO}

Diante do exposto, verificou-se que o trabalho pericial referente ao furto de energia elétrica em instalações residenciais deve ser precedido das seguintes etapas:

1. Verificação da habilitação legal do perito nomeado, junto ao conselho de classe;

2. Revisão da literatura e normas da ABNT atualizadas sobre a temática;

3. Simulação computacional para auxiliar os cálculos de consumo de energia, com a ressalva dos parâmetros de entrada e em especial da tipologia dos equipamentos;

4. Utilização de modelos matemáticos consagrados para previsão de consumo;

5. Análise processual;

6. Verificação do Termo de Ocorrência e Inspeção (TOI), de acordo com a Resolução ANEEL Nº 414/2010.

Desta forma, a presente cumpriu seu principal objetivo ao consolidar as principais informações sobre a Pericia Judicial em Instalações Elétricas Residenciais. Por fim, espera-se contribuir para o entendimento da temática e auxiliar no prosseguimento das etapas da perícia judicial na área em questão.

\section{REFERÊNCIAS}


AGÊNCIA NACIONAL DE ENERGIA ELÉTRICA (Brasil). Resolução Normativa 414/2010: atualizada até a REN 499/2012 / Agência Nacional de Energia Elétrica. Brasília: ANEEL, 2012.

ASSOCIAÇÃO BRASILEIRA DE NORMAS TÉCNICAS. NBR 5410: 2004. Instalações elétricas de baixa tensão. Rio de Janeiro, RJ: ABNT, 2005.

BRASIL. Lei Federal no 13.105/2015 - Código de Processo Civil. Disponível em: http://www.planalto.gov.br/ccivil_03/_ato2015-2018/2015/lei//13105.htm Acesso em: novembro de 2019.

Lei Federal no 6.496/1977 - Anotação de Responsabilidade Técnica. Disponível em: http://www.planalto.gov.br/ccivil_03/LEIS/L6496.htm Acesso em: novembro de 2019.

CREDER, Hélio. Instalações Elétricas. 15 ed. Rio de Janeiro: LTC, 2008.

FIKER, José. Perícias e Avaliações de Engenharia: Fundamentos Práticos. São Paulo, LEUD, 2011.

LIGHT. Regulamentação para fornecimento de energia elétrica a consumidores de baixa tensão (RECON-BT). Disponível em: http://www.light.com.br/Repositorio/Recon/RECON-BTCOMPLETO.pdf. Acesso em: novembro de 2019.

MAIA, Sérgio. Perícia em furto de energia elétrica - da inspeção administrativa ao exame pericial. Millennium. 2019.

MATERIAL DIDÁTICO. Curso de Especialização em Engenharia Elétrica - Da Entrada de Energia aos Sistemas de Proteção. Universidade Cândido Mendes, 2019.

MUNDO DA ELÉTRICA. Sistema de geração, transmissão, distribuição e consumo de energia para residências Disponível em: https://www.mundodaeletrica.com.br/um-pouco-mais-sobre-osistema-eletrico-de-potencia-sep/ Acesso em: novembro de 2019.

OMEGA TREINAMENTOS. Ligação de medidores até o quadro de distribuição. Disponível em: http://omegatreinamentos.coursify.me/courses. Acesso em: novembro de 2019.

SERTÃ JUNIOR, Luiz Roberto Charnaux. Perícia Judicial: Fundamentos, Ferramentas e Meio Ambiente. Rio de Janeiro, Processo, 2019. 